Bentham OPEN
CrossMark

\title{
Masquerading Orbital Sarcoidosis with Isolated Extraocular Muscle Involvement
}

\author{
Jane S. Kim ${ }^{1}$, Richard L. Scawn ${ }^{1}$, Bradford W. Lee ${ }^{1}$, Jonathan H. Lin ${ }^{1,2}$, Bobby S. Korn ${ }^{1,3}$ and Don \\ O. Kikkawa ${ }^{1,3, *}$
}

${ }^{I}$ Division of Ophthalmic Plastic and Reconstructive Surgery, Department of Ophthalmology, Shiley Eye Institute, University of California, San Diego, La Jolla, CA

${ }^{2}$ Ophthalmic Pathology Service, Department of Pathology, University of California, San Diego, La Jolla, CA

${ }^{3}$ Division of Plastic Surgery, Department of Surgery, University of California, San Diego, La Jolla, CA

\begin{abstract}
Two patients, previously diagnosed and treated for euthyroid, autoantibody-negative thyroid eye disease, presented with active orbitopathy. An atypical disease course and presentation prompted orbital biopsy. Extraocular muscle histopathology demonstrated noncaseating granulomatous inflammation consistent with presumed orbital sarcoidosis involving multiple extraocular muscles, including the inferior oblique in one of the cases. These two cases emphasize the importance of a broad differential diagnosis and the utility of an orbital biopsy in the context of an unusual disease presentation or poor treatment response. The patients' clinical course is discussed alongside important clinical signs, imaging findings, and biopsy results that support a diagnosis of isolated orbital sarcoidosis.
\end{abstract}

Keywords: Carotid-cavernous fistula, extraocular muscles, orbital sarcoidosis, orbital biopsy, thyroid eye disease.

\section{INTRODUCTION}

Sarcoidosis is an idiopathic multisystemic granulomatous disease that most commonly affects middle-aged AfricanAmerican females [1]. In addition to the lungs, lymph nodes, and skin, sarcoidosis frequently involves the eyes. Patients with ocular manifestations often present with a vague constellation of symptoms, including palpable mass, lid edema, proptosis, conjunctival erythema, and diplopia [2]. Although the most common ophthalmic manifestation in sarcoidosis is uveitis, infiltration of the adnexa, including the lacrimal glands, orbital fat, and extraocular muscles, can occur [1]. However, isolated adnexal involvement without systemic disease is rare and may be mistaken for another condition with similar clinical symptoms and radiographic findings, such as thyroid eye disease [1, 3]. We report two cases of presumed orbital sarcoidosis involving multiple extraocular muscles that were initially diagnosed as thyroid eye disease but were later reclassified as orbital sarcoidosis after orbital biopsies showed noncaseating granulomatous inflammation in the absence of systemic disease.

\section{CASE REPORTS}

\section{Case 1}

A 41 year old woman presented with painful proptosis and diplopia and was diagnosed with euthyroid, autoantibody-negative thyroid eye disease at an outside institution. The patient was treated with oral corticosteroids, orbital radiation, bilateral orbital decompression, rituximab, and mycophenolate mofetil over the course of a year. Despite treatment, her symptoms remained steroid-dependent. She presented to our institution for a second opinion.

\footnotetext{
* Address correspondence to this author at the Department of Ophthalmology, Shiley Eye Institute, University of California, San Diago, 9415 Campus Point Drive, La Jolla, CA 92093-0946, USA; E-mail: dkikkawa@ucsd.edu
} 
At presentation, she was taking $20 \mathrm{mg}$ of oral prednisone daily and $3 \mathrm{~g}$ of oral mycophenolate mofetil daily. Her general appearance was cushingoid. Visual acuity was 20/25 OU with correction. Extraocular movements were painful with mild limitation in all positions of gaze. Exophthalmometer readings were $21 \mathrm{~mm}$ OD and $22 \mathrm{~mm}$ OS. Although periorbital edema was present with increased resistance to retropulsion, eyelid retraction and lag were notably absent. Slit lamp and funduscopic examinations were unremarkable. Computed tomography (CT) of the orbits showed enlargement of multiple extraocular muscles with tendon involvement (Fig. 1A, B).
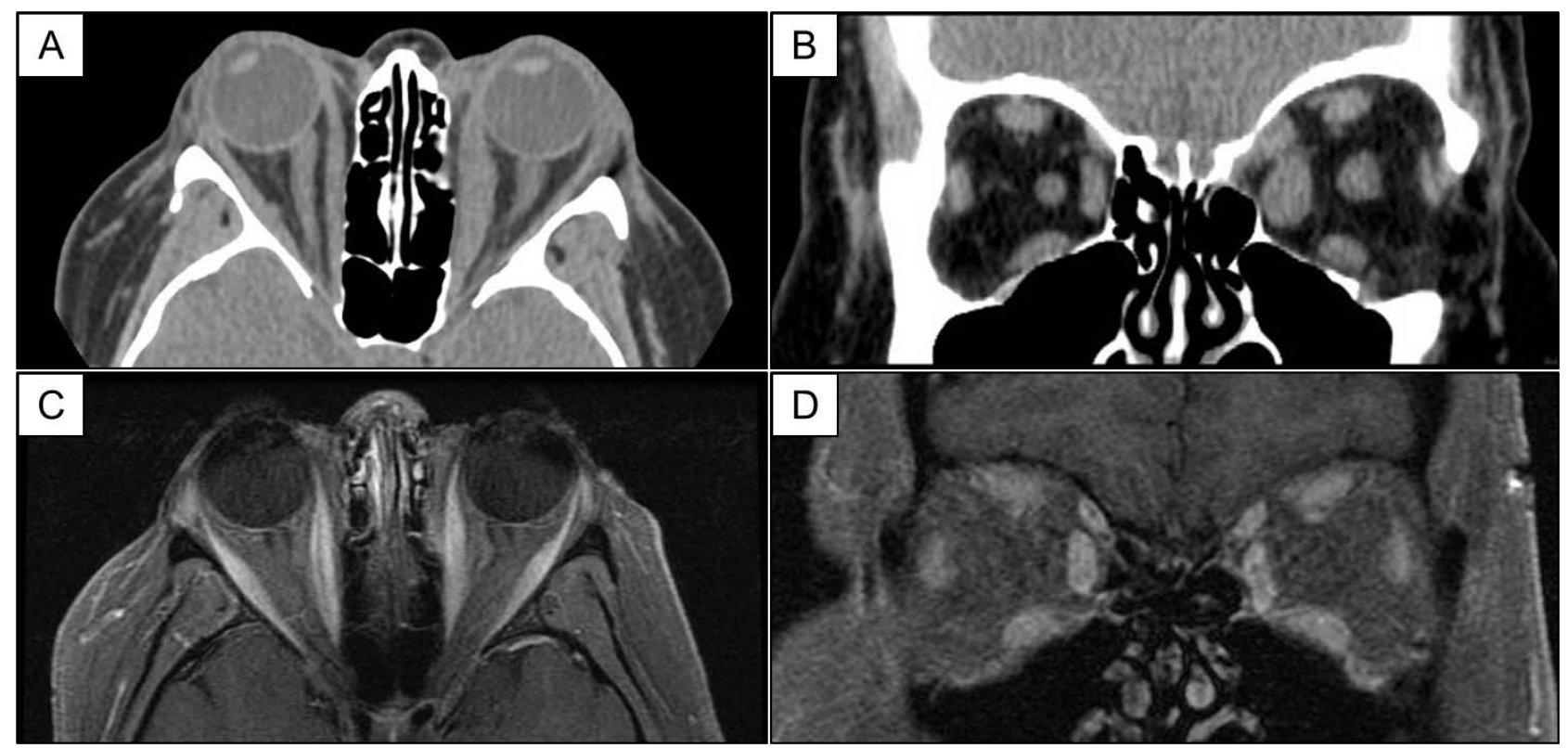

Fig. (1). Case 1. Axial (A) and coronal views (B) of CT imaging of the orbits show enlargement of multiple extraocular muscles, left greater than right, with tendon involvement. Eleven months post-diagnosis, axial (C) and coronal views (D) of T1-weighted, fatsuppressed MRI of the orbits demonstrate reduced extraocular muscle caliber with steroid-sparing immunosuppression.

In preparation for orbital biopsy, oral prednisone was tapered over one week with rebound orbital inflammation. Biopsy of the left inferior oblique, left medial rectus, left inferior rectus, and orbital fat was performed. Oral corticosteroids were resumed post-operatively.

Histopathology showed extensive inflammation and noncaseating granulomas without evidence of microorganisms or foreign bodies (Fig. 2). Rheumatology consultation was obtained. Systemic sarcoidosis screening, including chest radiograph, serum angiotensin-converting enzyme (ACE), and serum calcium, was negative. Serum levels of antineutrophil cytoplasmic antibodies (ANCAs), anti-nuclear antibodies, interferon-gamma release, and rapid plasma reagin (RPR) were within normal limits. A diagnosis of noncaseating granulomatous orbital myositis consistent with presumed isolated orbital sarcoidosis was made.

The patient was intolerant of methotrexate but was successfully switched to azathioprine as a steroid-sparing agent. Orbital inflammation remained controlled at an eleven month follow-up visit (Fig. 1C, D).

\section{Case 2}

A 71 year old woman with history of systemic hypertension, chronic obstructive pulmonary disease, and age-related macular degeneration presented with sudden periorbital edema and painful proptosis of both eyes. "Bulging" globes, headache, and decreased vision developed approximately two weeks after a prior fall. The patient was urgently referred to our institution by an outside ophthalmologist, who started the patient on brimonidine tartrate/timolol maleate ophthalmic solution for elevated intraocular pressure in both eyes.

On examination, visual acuity was 20/80 OD and 20/200 OS with correction. Color vision was reduced on the left, with two of eight color plates read correctly. Extraocular movements were largely intact, but eyelids were significantly swollen and erythematous. Eyelid retraction and lag were absent. Exophthalmometer readings were $27 \mathrm{~mm}$ OD and 26 $\mathrm{mm}$ OS with increased resistance to retropulsion but without palpable pulsations. Chemosis was also present in both eyes. Intraocular pressure was $16 \mathrm{~mm} \mathrm{Hg} \mathrm{OD} \mathrm{and} 20 \mathrm{~mm} \mathrm{Hg}$ OS on ocular hypertensive medication. Funduscopy 
revealed small macular drusen and hyperemic optic nerves with vascular congestion bilaterally.

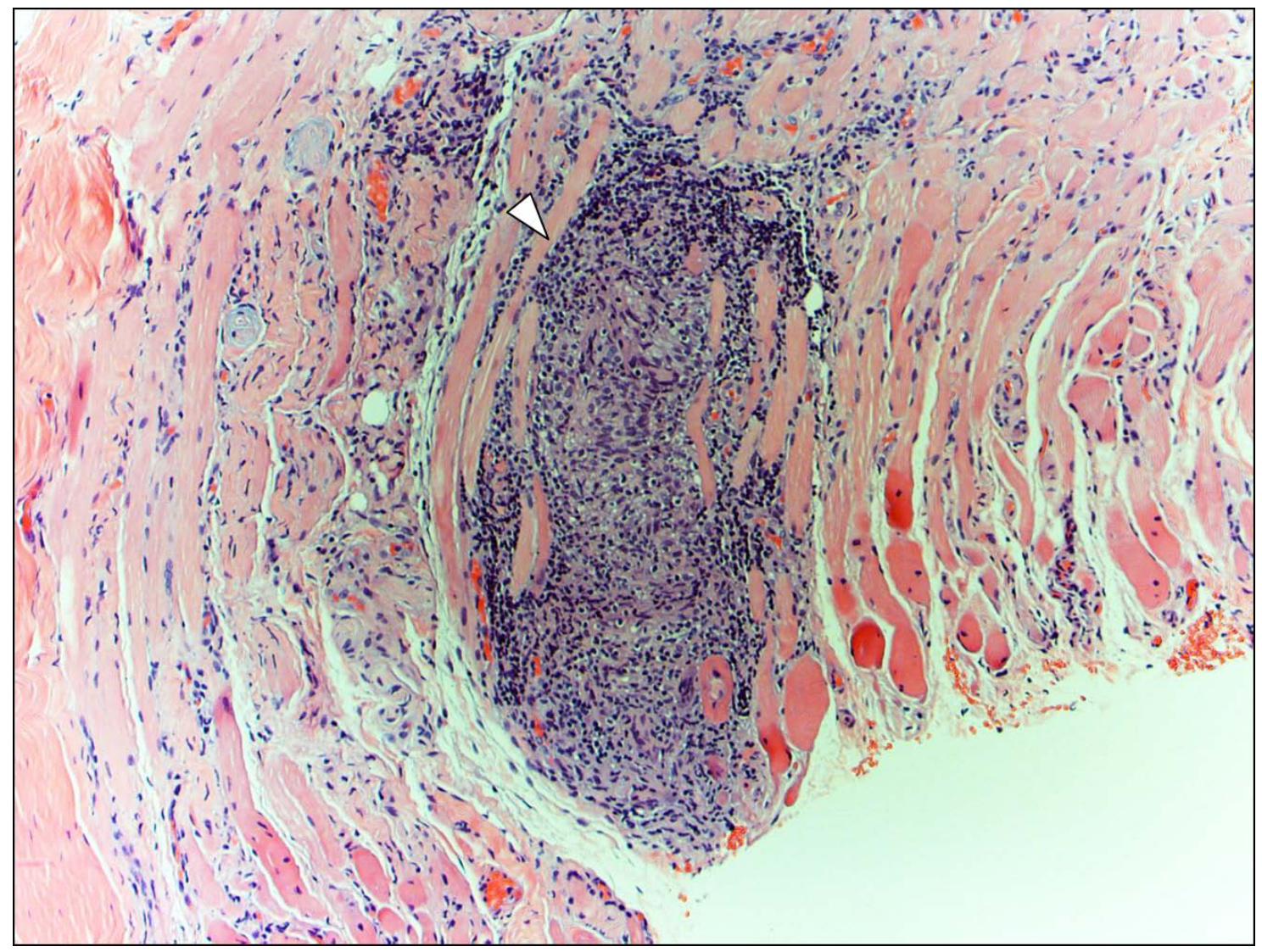

Fig. (2). Case 1. Hematoxylin-eosin staining of the inferior oblique muscle biopsy reveals a large noncaseating granuloma (arrowhead; magnification, 10x) in the absence of microorganisms (images not shown) and foreign bodies (image not shown), consistent with presumed orbital sarcoidosis.

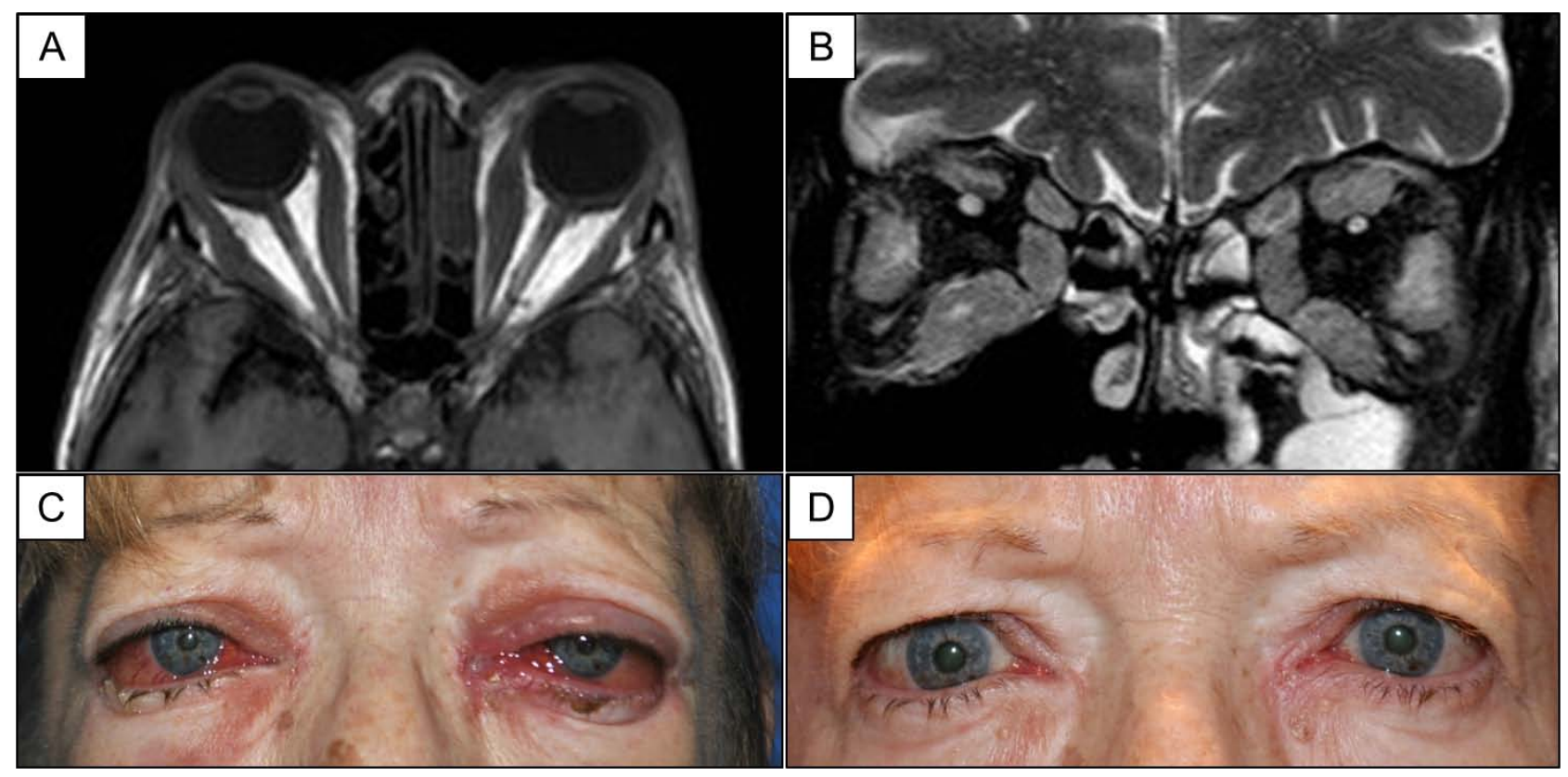

Fig. (3). Case 2. Axial (A) and coronal views (B) of T1-weighted MRI of the orbits show enlargement of multiple extraocular muscles with tendon involvement at the time of presentation. Clinical photographs before (C) and after (D) initiation of corticosteroid therapy show marked improvement in lid edema, chemosis, and conjunctival erythema. 
Given clinical suspicion of a carotid-cavernous fistula in the setting of recent head trauma, cerebral angiography was performed. No evidence of a direct or indirect carotid-cavernous fistula was found on cerebral angiogram. Magnetic resonance imaging (MRI) of the orbits revealed multiple enlarged extraocular muscles with tendon involvement bilaterally (Fig. 3A, B). Thyroid function was normal with normal serum levels of thyroid-stimulating immunoglobulin, anti-thyroglobulin antibodies, and anti-thyroid peroxidase antibodies. With a presumptive diagnosis of idiopathic orbital inflammation, the patient was started on $40 \mathrm{mg}$ of oral prednisone. The patient's pain, chemosis, and lid edema significantly improved with corticosteroids (Fig. 3C, D), but symptoms reappeared with steroid tapering.

Orbital biopsy was performed for a definitive diagnosis. Biopsy of the left lateral rectus muscle showed multiple noncaseating granulomas without evidence of microorganisms or foreign bodies (Fig. 4). Flow cytometric analysis was negative for lymphoma. Subsequent systemic sarcoidosis work-up, including chest radiograph, serum ACE, and serum calcium, was negative. Serum levels of ANCAs and RPR were within normal limits, and a tuberculin skin test was read as negative. The patient was diagnosed with noncaseating granulomatous myositis suggestive of orbital sarcoidosis in the absence of systemic features.

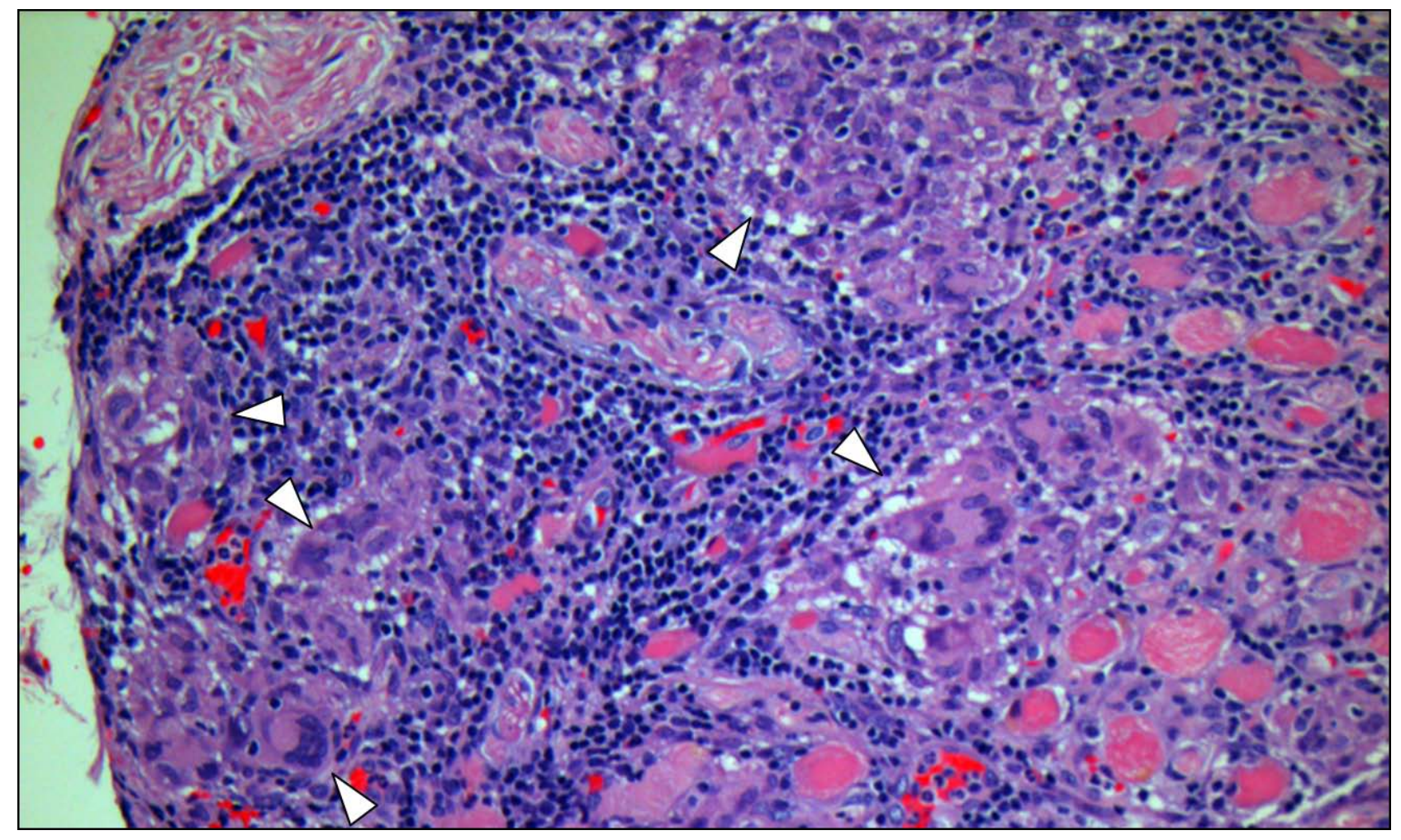

Fig. (4). Case 2. Hematoxylin-eosin staining of the lateral rectus muscle biopsy reveals multiple noncaseating granulomas (arrowheads; magnification, 20x) in the absence of microorganisms (images not shown) and foreign bodies (image not shown), strongly suggestive of orbital sarcoidosis.

The patient was referred to a rheumatologist, who recommended long-term treatment with low-dose oral prednisone for persistent symptoms. Because she experienced recurrent inflammatory symptoms with steroid tapering, the patient received 20 Gy of external orbital radiation and was able to discontinue corticosteroids two months after completion of radiotherapy.

\section{DISCUSSION}

Sarcoidosis is an idiopathic multisystemic granulomatous disease that most classically presents with symptoms of localized pulmonary disease and is diagnosed by the findings of hilar lymphadenopathy, elevated serum ACE, and possibly elevated serum calcium. However, none of these are requisite criteria for diagnosis, as sarcoidosis can only be definitively diagnosed by visualizing noncaseating granulomas on histology. Biopsy is not often immediately performed, and other systemic disease processes, such as granulomatosis with polyangiitis, tuberculosis, syphilis, and lymphoma, are effectively ruled out to establish a presumptive diagnosis. Although sarcoidosis is typically multi- 
systemic, isolated extraocular muscle involvement in the absence of systemic disease has been previously described [1, 3]. The two presented cases illustrate the varied manifestations of orbital sarcoidosis that can mimic other systemic conditions affecting the orbit as well as demonstrate the utility of orbital biopsy in establishing the diagnosis.

Isolated involvement of multiple extraocular muscles may have confounded the initial diagnosis of the two patients presented in this report, as extraocular muscle enlargement can occur in thyroid eye disease, sarcoidosis, Crohn's disease, granulomatosis with polyangiitis, primary infections, lymphoma, and other malignancies [4]. For example, thyroid eye disease can present with variable clinical signs and can be unilateral or bilateral. The classic combination of hyperthyroidism, eyelid retraction, exophthalmos, eyelid lag, restrictive extraocular movements, and optic neuropathy is relatively rare, with just $5 \%$ of patients exhibiting all of these signs [5].

Thyroid eye disease most frequently involves the inferior rectus, followed by the medial rectus, superior rectus, lateral rectus, and less frequently the superior and inferior oblique [6]. Thyroid eye disease also characteristically spares the muscle tendons, in contrast to the radiographic findings in our two cases (Figs. 1A, 3A). Although tendon involvement has been described in association with thyroid eye disease [7], any extraocular muscle enlargement that extends to the tendons and includes the inferior oblique should prompt consideration of an alternative diagnosis.

Orbital biopsy is indicated when orbital pathology is either undiagnosed or atypical in presentation or treatment response. Given its high degree of accessibility and low rate of adverse events, the inferior oblique has been described as the "muscle of choice" for orbital biopsies [8]. Biopsies are most useful when directed at abnormal tissue. In the first case, biopsy of the inferior oblique, lateral and inferior recti, and orbital fat was performed. Multiple biopsies with cessation of immunosuppression were performed to increase diagnostic yield despite long-term, disease-modifying immunosuppressive treatment. For the second case, we chose to biopsy the lateral rectus in combination with lateral wall removal for symptomatic improvement. Although both patients developed transient worsening of clinical symptoms with planned steroid tapering, a definitive diagnosis was achieved with good clinical response to steroidsparing immunosuppression in the first case and orbital radiation in the second.

Isolated orbital sarcoidosis may be the first manifestation of systemic sarcoidosis, and guidelines suggest that patients with isolated orbital sarcoid infiltration should be initially screened for systemic disease and followed clinically [9]. These two cases demonstrate that orbital sarcoidosis can affect multiple extraocular muscles, including the inferior oblique, and emphasize that expanding the differential diagnosis and performing an orbital biopsy are important when there is an unusual clinical presentation or poor treatment response.

\section{CONFLICT OF INTEREST}

The authors confirm that this article content has no conflict of interest.

\section{ACKNOWLEDGEMENTS}

Generously supported by Steve and Kathleen Flynn; Bell Charitable Foundation; Research to Prevent Blindness; and UC San Diego Vision Research Center Core Grant P30EY022589.

\section{REFERENCES}

[1] Obenauf CD, Shaw HE, Sydnor CF, Klintworth GK. Sarcoidosis and its ophthalmic manifestations. Am J Ophthalmol 1978; 86(5): 648-55. [http://dx.doi.org/10.1016/0002-9394(78)90184-8] [PMID: 568886]

[2] Mavrikakis I, Rootman J. Diverse clinical presentations of orbital sarcoid. Am J Ophthalmol 2007; 144(5): 769-75. [http://dx.doi.org/10.1016/j.ajo.2007.07.019] [PMID: 17869205]

[3] Cornblath WT, Elner V, Rolfe M. Extraocular muscle involvement in sarcoidosis. Ophthalmology 1993; 100(4): 501-5. [http://dx.doi.org/10.1016/S0161-6420(93)31615-5] [PMID: 8479707]

[4] Lacey B, Chang W, Rootman J. Nonthyroid causes of extraocular muscle disease. Surv Ophthalmol 1999; 44(3): 187-213. [http://dx.doi.org/10.1016/S0039-6257(99)00101-0] [PMID: 10588439]

[5] Bartley GB, Fatourechi V, Kadrmas EF, et al. Clinical features of Graves' ophthalmopathy in an incidence cohort. Am J Ophthalmol 1996; 121(3): 284-90.

[http://dx.doi.org/10.1016/S0002-9394(14)70276-4] [PMID: 8597271]

[6] Kakizaki H, Zako M, Iwaki M. Thyroid-associated inferior oblique myopathy. Ophthalmology 2007; $114(11): 2106$. [http://dx.doi.org/10.1016/j.ophtha.2007.04.034] [PMID: 17980760]

[7] Ben Simon GJ, Syed HM, Douglas R, McCann JD, Goldberg RA. Extraocular muscle enlargement with tendon involvement in thyroidassociated orbitopathy. Am J Ophthalmol 2004; 137(6): 1145-7. 
[http://dx.doi.org/10.1016/j.ajo.2004.01.033] [PMID: 15183812]

[8] Hoogenraad TU, Tan KE, Eelderink HJ, Veldman H, Jennekens FG. The inferior oblique as muscle of choice for biopsies of extraocular muscles. Br J Ophthalmol 1979; 63(10): 680-3.

[http://dx.doi.org/10.1136/bjo.63.10.680] [PMID: 508677]

[9] American Thoracic Society. Statement on sarcoidosis. Am J Respir Crit Care Med 1999; 160(2): 736-55. [http://dx.doi.org/10.1164/ajrccm.160.2.ats4-99] [PMID: 10430755]

(C) Kim et al.; Licensee Bentham Open.

This is an open access article licensed under the terms of the Creative Commons Attribution-Non-Commercial 4.0 International Public License (CC BY-NC 4.0) (https://creativecommons.org/licenses/by-nc/4.0/legalcode), which permits unrestricted, non-commercial use, distribution and reproduction in any medium, provided the work is properly cited. 